\title{
Occupational Exposure to Blood and Body Fluids among Interns in a Tertiary Hospital in Port Harcourt, Nigeria
}

\author{
Nsirimobu Ichendu Paul*, Tamunopreye Jaja, Peace Ibo Opara \\ Department of Paediatrics, University of Port Harcourt Teaching Hospital, Port Harcourt, Nigeria \\ Email: *nsirimobu.paul@yahoo.com
}

How to cite this paper: Paul, N.I., Jaja, T. and Opara, P.I. (2018) Occupational Exposure to Blood and Body Fluids among Interns in a Tertiary Hospital in Port Harcourt, Nigeria. Open Access Library Journal, 5: e4122.

https://doi.org/10.4236/oalib.1104122

Received: November 6, 2017

Accepted: April 10, 2018

Published: April 13, 2018

Copyright $\odot 2018$ by authors and Open Access Library Inc.

This work is licensed under the Creative Commons Attribution International License (CC BY 4.0).

http://creativecommons.org/licenses/by/4.0/

\section{(c) (i) Open Access}

\begin{abstract}
Background: Health care workers especially interns may be at increased health risk due to exposure to blood and body fluids. Objective: To determine the prevalence of occupational exposure to blood and body fluids (EBBF) among interns at the University of Port Harcourt Teaching hospital (UPTH). Methodology: This cross-sectional study was carried out among interns at the UPTH. Informed written consent was obtained. The obtained data from a self-administered questionnaire and Infection Control Team records were analysed using SPSS version 21 and are presented as prose and tables. Results: Eighty four interns were studied giving a response rate of 93.3\%: 40 (47.6\%) were males while $44(52.4 \%)$ were females giving a male:female ratio of 1:1.1. Thirty two (38.1\%) were aware of the availability of sharp bins, $55(65.5 \%)$ recapped and discarded into waste bins while $4(4.8 \%)$ discarded into sharp bins without re-capping. Prevalence of EBBF was $89.3 \%$. Thirty one (41.4\%) had Blood and Body Fluid Splash (BBFS), 22 (29.3\%) had Needle Stick Injury (NSI) while $22(29.3 \%)$ had combined NSI and BBFS. Thirteen $(29.5 \%)$ of the NSI occurred during rotation in Paediatrics. Seventeen (32.1\%) and 16 (30.2\%) of BBFS occurred during Obstetrics and Gynaecology and Paediatrics rotation respectively. Eleven (25\%) reported the NSI to the Infection control team (ICT) and 3 (27.3\%) completed 28 days of Highly Active Anti-Retroviral Therapy (HAART). Common reason for not reporting was not been aware of the Infection Control Team (ICT) in 10 (30.3\%). Conclusion: There is a need for improved training and close supervision of interns.
\end{abstract}

\section{Subject Areas}

Hematology 


\section{Keywords}

Occupational Exposure, Interns, UPTH

\section{Introduction}

Healthcare workers (HCWs) are exposed to hazards from contact with blood and body fluids of patients in their daily course of work. Needle stick injuries (NSIs) and cuts are the common accidents that expose HCWs to blood and body fluids [1] [2] and they usually result from unsafe practices such as recapping of needles, manipulating used needles such as bending, breaking, or cutting hypodermic needles, and passing of needles from one HCW to another. These preventable injuries expose $\mathrm{HCW}$ to over 20 different pathogens [3] the most common being HIV, Hepatitis B and Hepatitis C [4]. In 2002, the World Health Organization (WHO) reported that 2 million HCWs experience percutaneous exposure to infectious diseases and $39 \%$ of hepatitis C virus (HCV), $37.6 \%$ of hepatitis B virus (HBV) and $4.4 \%$ of HIV/AIDS in HCWs worldwide are due to NSIs [5].

The risk of exposure to blood and body fluids by HCWs varies among the different health workers, the different sections of the hospital and from one type of procedure to the other. A cross-sectional study of HCWs in New Delhi, India revealed that the incidence of exposure to potential infectious material was highest among the staff nurses at $39.63 \%$, followed by interns at $37.34 \%$, technicians at $26.92 \%$ and least among the resident doctors at $21.01 \%$ [2]. Erhabor et al. [6] in Port Harcourt, Nigeria found that exposures were more in the Paediatric Unit followed by the Phlebotomy Section of the Laboratory Unit and Surgery. Singru and Banerjee found that re-capping of needles was the most hazardous procedure particularly among interns and staff nurses, while suturing was the commonest procedure among the resident doctors [2]. However, Mbaisi et al. [7] in Kenya reported that suturing was the most common procedure during which injuries occurred, followed by blood specimen collection and handling of IV lines.

Interns are doctors in the first year after qualification, who undergo a twelve-month rotation in the four key departments of Medicine. They spend three months in each of the departments and are supervised by certified consultants who eventually certify them when their performance is satisfactory based on recommended criteria. Interns may be at a higher risk of being exposed to blood and body fluids as they are learning to carry out procedures and may be less cautious than other health workers. They are also less likely to practice universal precautions and are more likely to sustain needle stick injuries due to inexperience. The present study was carried out to determine the prevalence of blood and body fluid exposures among interns at the UPTH, knowledge and practice of sharp disposal methods, the common type of exposure, the section of 
the hospital with the highest risk and post-exposure actions taken by these interns.

\section{Methodology}

This was a cross sectional study carried out from $1^{\text {st }}-30^{\text {th }}$ June 2015 among intern doctors (House officers) at the University of Port Harcourt Teaching Hospital. All interns who had done at least nine Months out of the required twelve Months posting and were at their $4^{\text {th }}$ (last) rotation were included in the study. Informed written consent was obtained and only those who gave consent completed the questionnaire. Data collection was done using a structured self-administered questionnaire while the Infection Control Team (ICT) record was used to confirm reported cases. The questionnaire contained information on the demographic characteristics of the interns, availability of sharp bins and its routine usage, the forms of exposure to blood and body fluids, the department and unit where the exposure occurred, and the actions taken by the interns following the exposure. The obtained data was entered and analysed using SPSS version 21 and is presented as prose and tables.

\section{Results}

\subsection{Response Rate and Sex Distribution of the Study Population}

A total of Ninety questionnaires was administered and eighty four were returned giving a response rate of $93.3 \%$. Of these, $40(47.6 \%)$ were males while 44 (52.4\%) were females giving a male: female ratio of 1:1.1. Eighty three (98.8\%) were graduates from 16 Nigerian Medical schools and 1 (1.2\%) was an International graduate (Table 1 ).

\subsection{Awareness of Availability of Sharp Bins and Routine Practice with Sharps}

All the wards had sharp bins but only $32(38.1 \%)$ were aware of the availability of sharp bins in the wards, $46(54.8 \%)$ said there were no sharp bins while 6 (7.2\%) were not aware. Fifty five (65.5\%) of the interns recapped their sharps and discard into waste bins while only $4(4.8 \%)$ discard the sharps into sharp bins without re-capping (Table 2).

Table 1. Sex distribution and medical school attended by interns.

\begin{tabular}{ccc}
\hline Sex & Frequency & Percent \\
\hline Male & 40 & 47.6 \\
Female & 44 & 52.4 \\
Total & 84 & 100.0 \\
Medical school attended & Frequency & Percent \\
Nigerian & 83 & 98.8 \\
Foreign & 1 & 1.2 \\
Total & 84 & 100.0 \\
\hline
\end{tabular}


Table 2. Routine practice with sharps.

\begin{tabular}{ccc}
\hline Routine practice with sharps & Frequency & Percent (\%) \\
\hline Recap needle and discard into waste bin (a) & 55 & 65.5 \\
Recap needle and discard into sharp bin (b) & 11 & 13.1 \\
Discard sharps into sharp bin without recapping (c) & 4 & 4.8 \\
Discard sharps in the waste bin without recapping (d) & 2 & 2.4 \\
Scoop the cap and discard both (e) & 1 & 1.2 \\
$\mathrm{a}+\mathrm{b}^{*}$ & 6 & 7.1 \\
$\mathrm{a}+\mathrm{c}^{*}$ & 1 & 1.2 \\
$\mathrm{~b}+\mathrm{c}^{*}$ & 2 & 2.4 \\
$\mathrm{c}+\mathrm{d}^{*}$ & 1 & 1.2 \\
$\mathrm{a}+\mathrm{b}+\mathrm{c}+\mathrm{d}^{*}$ & 1 & 1.2 \\
Total & 84 & 100.0 \\
\hline
\end{tabular}

${ }^{*}$ combination of these practices.

\subsection{Prevalence of Exposure to Blood and Body Fluids}

Seventy five of the 84 interns had at least one exposure to blood and body fluid giving a prevalence rate of $89.3 \%$. Of these, 31 (41.4\%) had BBFS, 22 (29.3\%) had NSI while $22(29.3 \%)$ had combined NSI and BBFS (total no of those exposed to BBFS is 53 while that of NSI is 44 ).

\subsection{Rotation Where NSI and BBFS Occurred}

Of the 44 NSI, 13 (29.5\%) occurred during rotation in Paediatrics while 11 (25.0\%) occurred in Internal Medicine. Also, 17 (32.1\%) of BBFS occurred on Obstetrics and Gynaecology (O \& G) while 16 (30.2\%) occurred during Paediatric rotation (Table 3 ).

\subsection{Site of NSI and BBFS Exposure}

Nearly a third of NSI occurred in the Accident and Emergency (29.5\%) and the General ward (27.3\%) respectively while 13 (24.5\%) of the BBFS occurred in the Children's Emergency Ward (CHEW) and the Labour ward (L. Ward) (Table 4).

N/B: G. Ward-General Ward, CHOP_Children Outpatient; SCBU-Special Care Baby Unit.

\subsection{Notification to and Response by the ICT}

Out of the 44 interns who had NSI, 11 (25\%) reported to the Infection control team (ICT) while 33 (75\%) did not report. Of those who reported to the ICT, 7 (63.6\%) reported within $30 \mathrm{mins}, 3(27.3 \%)$ within one hour while 1 (9.1\%) reported between 1 - 6 hrs. For those that reported to the ICT, 7 (63.6\%) had HIV screening done for them and the patient, 3 (27.3\%) commenced ARV after HIV screening 
was done for them and patient while in $1(9.1 \%)$ only counselling was done.

\subsection{Reason for Non-Notification}

Ten (30.3\%) of those that did not report said they were not aware of the Infection Control Team (ICT) while 9 (27.3\%) said that the source patient was HIV negative and so they did not think it was important (Table 5).

Among those who had NSI, 19 (43.2\%) washed the site immediately with soap and water and applied antiseptic, $14(31.8 \%)$ washed the site immediately with soap and water only, 3 (6.8\%) allowed site to bleed for a while before washing with soap and water, $1(2.3 \%)$ washed with sodium hypochlorite solution (JIK) while 7 (15.9\%) did nothing at all.

\subsection{Orientation on Universal Precaution and Knowledge of PEP}

Among the interns, 54 (64.3\%) did not have orientation on universal precaution while $30(35.7 \%)$ did. Nineteen $(22.6 \%)$ were aware of the existence of a protocol for the management of exposure to blood and body fluids, 14 (16.7\%) said there was no protocol in existence while 49 (58.3\%) were not aware if there was or not. Of the nineteen who were aware of a hospital protocol only 5 (26.3\%) could remember a point on the list.

Table 3. Rotation where NSI and BBFS.

\begin{tabular}{cccccccc}
\hline Rotation & Paediatrics & $\begin{array}{c}\text { Internal } \\
\text { Medicine }\end{array}$ & O \& G & A \& E $E^{* *}$ & $\begin{array}{c}\text { General } \\
\text { Surgery }\end{array}$ & Ophthalmology & Total \\
\hline NSI (\%) & $13(29.5)$ & $11(25.0)$ & $9(20.5)$ & $6(13.6)$ & $4(9.1)$ & $1(2.3)$ & $44(100.0)$ \\
BBFS (\%) & $16(30.2)$ & $10(18.9)$ & $17(32.1)$ & $3(5.6)$ & $5(9.4)$ & $2(3.8)$ & $53(100.0)$
\end{tabular}

NSI-Needle Stick Injury; BBFS—Blood and Body Fluid Splash. ${ }^{* *}$ Accident and Emergency.

Table 4. Site of NSI and BBFS.

\begin{tabular}{ccccccccc}
\hline Site & A\&E & CHEW & G. Ward & L. Ward & Theatre & CHOP & SCBU & Total \\
\hline NSI (\%) & $13(29.5)$ & $10(22.7)$ & $12(27.3)$ & $3(6.8)$ & $3(6.8)$ & $2(4.6)$ & $1(2.3)$ & $44(100.0)$ \\
BBFS (\%) & $7(13.2)$ & $13(24.5)$ & $12(22.6)$ & $13(24.5)$ & $5(9.4)$ & $2(3.8)$ & $1(2.0)$ & $53(100.0)$ \\
\hline
\end{tabular}

NSI-Needle Stick Injury; BBFS-Blood and Body Fluid Splash. N/B: G. Ward-General Ward CHOP_Children Outpatient; SCBU_Special Care Baby Unit, L. Ward-Labour Ward.

Table 5. Reason for not reporting to the IC.

\begin{tabular}{ccc}
\hline Reason for not reporting & Frequency & Percent (\%) \\
\hline Not aware of the ICT & 10 & 30.3 \\
Didn't think it was important Source patient was HIV negative & 9 & 27.3 \\
pray and leave it to God & 5 & 15.1 \\
No reason & 4 & 12.1 \\
Pricked with an unused needle & 3 & 9.1 \\
Forgot to report & 2 & 6.1 \\
Total & 33 & 100.0 \\
\hline
\end{tabular}

Action taken by the subjects following Exposure to blood and body fluids. 


\section{Discussion}

The study shows that the level of awareness of the availability of sharp bins and its routine usage was low. Only $4.8 \%$ knew and practiced correctly the disposal of sharps while over $75 \%$ still recap sharps before discarding them. It is amazing that interns were still recapping needles; a practice that should be discouraged as sharp bins are provided for disposal of needles and sharp objects in the hospital. Knowledge of the availability of sharp bins and proper use of sharps must be ensured at all times by managers and supervisors of all HCWs as this does not only limit self-occupational hazards but also that to co-workers. According to standard principles for the safe use and disposal of sharps [8], all used sharps: must not be bent or broken before disposal, must not be recapped, used sharps must be discarded immediately into a sharps container that conforms to current standard by the person generating the sharps waste. However, in Dentistry, if recapping or disassembly is unavoidable, a risk assessment must be undertaken and appropriate safety devices should be used. Making a suitable and sufficient assessment of risks involves identifying the hazards (something with the potential to do harm), and evaluating the extent of risks (the likelihood that the harm from a particular hazard is realized); and identifying measures needed to comply with legal requirements [8].

The prevalence of at least a single exposure to blood and body fluid among interns at the University of Port Harcourt Teaching Hospital was $89.3 \%$. Though Clarke et al., [9] found that the probability of ever having a needle-stick injury was inversely related to years of experience, this high exposure rate compared to many studies [2] [10] [11] [12], probably shows a fundamental deficiency in knowledge and practice of universal precaution among these interns. However, these other studies were done among different HCWs and so focusing this study on only interns may also contribute to this high prevalence. Interns we know are in-experienced and are learning and acquiring new skills and so may be prone to increased exposure to blood and body fluids but this magnitude of exposure is rather worrisome. These are interns who are pooled from different training institutions in Nigeria so it's unlikely that this problem is peculiar to UPTH. Could a fundamental deficit in training or supervision of these interns also have contributed to this prevalence? Similar studies from other centres are needed to elucidate this. However, this problem is not peculiar to us, in a study in Newcastle provincial Hospital South Africa among forty nine HCW a similar prevalence of $84 \%$ was reported [13]. If this large number of HCW are exposed to occupational hazards and considering the prevalence of these blood borne infections especially in sub-Saharan African this portends great danger for many HCWs if there is no change in its trend.

Unlike in some other studies [2] [10] [11], BBFS constituted more of the occupational exposure compared to NSI and more of the NSI took place during Paediatric rotation in the Accident and Emergency, General Ward and Children Emergency Ward (CHEW). The BBFS was more prevalent during Obstetrics and 
Gynaecology rotation occurring more in the CHEW and Labour ward. This findings support a previous study in Port Harcourt by Erhabor et al. [6] that most of the exposure to blood and body fluids was in Paediatrics and this may be due to the similarity in the study area. However, report from a China study [14] showed that the subjects with the highest NSI were from departments of gynaecology and obstetrics, surgical departments, intensive care units and emergency rooms. This study was however carried out among resident doctors.

In this study, only $25 \%$ of those with NSI reported to the ICT and this is similar to findings from other studies [2] [11] [15]. The primary reasons for not reporting were that they were not aware of an Infectious Control Team and that the source patient was HIV negative. Unreported NSI are a serious problem and prevent injured HCWs from receiving post exposure prophylaxis against HIV, which has been shown to be $80 \%$ effective against HIV infection [16]. It also limits close surveillance to other blood borne pathogens and to HIV infection for those that the source patient is in their window period. Where workers compensation benefits exist, lack of documentation of the injury will prevent the HCW from benefiting from it should he or she become infected with HIV or other blood-borne pathogens [16]. Those who reported had proper post exposure measures taken by the Infection Control Team and $27.3 \%$ of them commenced and completed 28 day ARV as post exposure prophylaxis.

The immediate actions taken by all except $15.7 \%$ of those who had NSI were according to stipulated guidelines. These $15.9 \%$ are among those who said that the source patient was HIV negative and that the injury was inflicted with an unused needle. Accidental Inoculation procedures guideline [17] stipulates that, if one is exposed to blood, serum or other body fluids: Immediate Action to be initiated within less than one minute is to wash the wound with soap and water (not scrubbed), dry and cover with a dressing. Splashes to non-intact skin should be cleaned liberally with water while splashes into eyes, mouth and other mucous membranes should be irrigated with copious amounts of clean water. If contact lenses are worn eye irrigation should take place before and after removing lenses. However, some authorities stipulate that one should encourage the injury to bleed gently before washing with soap and water [18]. The guideline also stipulates that in the absence of clean water, one should wash with an available antiseptic [17] [18].

At the UPTH, newly employed Interns undergo a two day hospital orientation before commencement of duties. It is here that among other topics universal safety precaution is marshalled by the hospital ICT, which is to be re-enforced at the departmental and unit level. Unfortunately, 54 (64.3\%) of these interns failed to go through this process. This may be contributory to the high prevalence rate in this study and the lack of awareness of an existing hospital protocol for the management of exposure to blood and body fluid.

\section{Conclusions}

In conclusion, this study has shown that the prevalence of exposure to blood and 
body fluid among interns at the UPTH is high and that there exist lacunae in knowledge on occupational hazard preventive measures that must be filled.

We hereby recommend that:

1) A written policy on universal precaution with emphasis on the proper use of sharps, availability of sharp bins and its usage to be conspicuously displayed in all hospital wards.

2) A proper reporting protocol following accidental NSI and BBFS should be made known to all hospital staff.

3) A protocol on PEP should also be made readily available in all the wards and routinely communicated to all HCWs especially medical students and interns.

4) Regular training and retraining of medical students and interns on invasive procedures involving the use of sharps.

5) Close supervision of interns especially when carrying out procedures that may expose them to blood and body fluids.

\section{Limitation of the Study}

The fact that the collected data was based on past incidents (previous nine Months) there may be over or under reporting of the exposures.

Also, Limited funding restricted this study to the study centre, otherwise a comparative analysis of the exposure of these interns with those in other secondary and tertiary care centres in the same Port Harcourt city may have broadened the scope of the recommendation.

\section{Conflict of Interest}

The authors wish to state that the article is original and there is no conflict of interest.

\section{Financial Support}

The article is self-sponsored by the authors.

\section{References}

[1] World Health Organization (2002) Protecting Health-Care Workers- Preventing Needlestick Injuries. WHO World Health Report, WHIO, Geneva.

[2] Singru, S.A. and Banerjee, A. (2008) Occupational Exposure to Blood and Body Fluids among Health Care Workers in a Teaching Hospital in Mumbai, India. Indian Journal of Community Medicine, 33, 26-30. https://doi.org/10.4103/0970-0218.39239

[3] Centers for Disease Control and Prevention (1998) Guidelines for Infection Control in Health Care Personnel. Infection Control \& Hospital Epidemiology, 19, 445.

[4] International Health Care Worker Safety Center (1998) Estimated Annual Number of U.S. Occupational Percutaneous Injuries and Mucocutaneous Exposures to Blood or Potentially Infective Biological Substances. Advances in Exposure Prevention, 4, 3.

[5] World Health Organization (2002) Needle Stick Injury. 
http://www.who.int/occupational_health/topics/needinjuries/en/

[6] Erhabor, O., Ejele, O.A. and Nwauche, C.A. (2007) Epidemiology and Management of Occupational Exposure to Blood Borne Viral Infections in a Resource Poor Setting: The Case for Availability of Post Exposure Prophylaxis. Nigerian Journal of Clinical Practice, 10, 100-104.

[7] Mbaisi, E.M., Ng'ang'a, Z., Wanzala, P. and Omolo, J. (2013) Prevalence and Factors Associated with Percutaneous Injuries and Splash Exposures among Health-Care Workers in a Provincial Hospital, Kenya. Pan African Medical Journal, 14, 10. https://doi.org/10.11604/pamj.2013.14.10.1373

[8] (2012) Standard Principles for the Safe Use and Disposal of Sharps. Infection: Prevention and Control of Healthcare-Associated Infections in Primary and Community Care: Partial Update of NICE Clinical Guideline, 2, 98-103. https://www.ncbi.nlm.nih.gov/pubmedhealth/PMH0050779/

[9] Clarke, S.P., Rockett, J.L., Sloane, D.M. and Aiken, L.H. (2002) Organizational Climate, Staffing and Safety Equipment as Predictors of Needlestick Injuries and Near Misses in Hospital Nurses. American Journal of Infection Control, 30, 207-216. https://doi.org/10.1067/mic.2002.123392

[10] Newsom, D.H. and Kiwanuka, J.P. (2002) Needle-Stick Injuries in an Ugandan Teaching Hospital. Annals of Tropical Medicine \& Parasitology, 96, 517-522. https://doi.org/10.1179/000349802125001186

[11] Resnic, F. and Noerdlinger, M.A. (1995) Occupational Exposure among Medical Students and House Staff at a New York City Medical Center. Archives of Internal Medicine, 155, 75-80. https://doi.org/10.1001/archinte.1995.00430010081011

[12] Newsom, D.H. and Kiwanuka, J.P. (2002) Needle-Stick Injuries in an Ugandan Teaching Hospital. Annals of Tropical Medicine \& Parasitology, 96, 517-522. https://doi.org/10.1179/000349802125001186

[13] Adefolalu, A.O. (2014) Needle Stick Injuries and Health Workers: A Preventable Menace. Annals of Medical and Health Sciences Research, 4, 159-160. https://doi.org/10.4103/2141-9248.138046

[14] Shi, C.L., Zhang, M. and Xie, C. (2011) Study on Status of Needle-Stick and Other Sharps Injuries among Healthcare Workers in a General Hospital. Zhonghua Lao Dong Wei Sheng Zhi Ye Bing Za Zhi, 29, 939-943. [In Chinese]

[15] Cvejanov-Kezunović, L., Mustajbegović, J., Milošević, M. and Čivljak, R. (2014) Occupational Exposure to Blood among Hospital Workers in Montenegro. Archives of Industrial Hygiene and Toxicology, 65, 273-280. https://doi.org/10.2478/10004-1254-65-2014-2493

[16] Rahul, S., Rasania, S.K., Anita, V. and Saudan, S. (2010) Study of Prevalence and Response to Needlestick Injuries among Health Care Workers in a Tertiary Care Hospital in Delhi, India. Indian Journal of Community Medicine, 35, 74-77.

[17] Centres for Disease Prevention and Control (2018) Bloodborne Infectious Diseases: HIV/AIDS, Hepatitis B, Hepatitis C: Emergency Needlestick Information.

[18] Health and Safety Services (2014) Managing Exposure to Blood and Body Fluids. University of Hull Booklet, Issue 3. 